\title{
Effective Scheme of Channel Tracking and Estimation for Mobile WiMAX DL-PUSC System
}

\author{
Phuong Thi Thu Pham ${ }^{1}$ and Tomohisa Wada ${ }^{1,2}$ \\ ${ }^{1}$ Information Engineering Department, Graduate School of Engineering and Science, University of The Ryukyus, \\ 1 Senbaru, Nishihara, Okinawa 903-0213, Japan \\ ${ }^{2}$ Magna Design Net, Inc., L1831-1 Oroku, Naha-city, Okinawa 901-0155, Japan
}

Correspondence should be addressed to Phuong Thi Thu Pham, thuphuong@lsi.ie.u-ryukyu.ac.jp

Received 28 September 2009; Accepted 30 November 2009

Academic Editor: Rashid Saeed

Copyright ( $) 2010$ P. T. T. Pham and T. Wada. This is an open access article distributed under the Creative Commons Attribution License, which permits unrestricted use, distribution, and reproduction in any medium, provided the original work is properly cited.

This paper introduces an effective joint scheme of channel estimation and tracking for downlink partial usage of subchannel (DLPUSC) mode of mobile WiMAX system. Based on the pilot pattern of this particular system, some channel estimation methods including conventional interpolations and a more favorable least-squares line fitting (LSLF) technique are comparatively studied. Besides, channel estimation performance can be remarkably improved by taking advantage of channel tracking derived from the preamble symbol. System performances in terms of packet error rate (PER) and user link throughput are investigated in various channels adopted from the well-known ITU models for mobile environments. Simulation results show a significant performance enhancement when the proposed joint scheme is utilized, at least $5 \mathrm{~dB}$, compared to only commonly used channel estimation approaches.

\section{Introduction}

Wireless metropolitan area network (Wireless MAN) or worldwide interoperability for microwave access (WiMAX), which is defined in IEEE Std. $802.16 \mathrm{~d} / \mathrm{e}[1,2]$, is a new technology that provides wireless access in fixed and mobile environments. Some modes in this system utilize orthogonal frequency division multiple access (OFDMA) technique as a modulation method. This technique is adopted from the powerful orthogonal frequency division multiplexing (OFDM) which effectively mitigates the impairment of the time-variant frequency selective fading channel [3, 4]. A typical OFDM system is shown in Figure 1.

DL-PUSC, as specified in [1], is one of the multiple access modes for downlink direction which is popularly used for performance analysis. This scheme divides OFDM symbol into subchannels and assigns them to users/subscribers. Each subchannel is further partitioned into groups of 14 consecutive subcarriers called clusters. Clusters of one user are not continuously connected but are pseudorandomly permuted over OFDM symbol among users so that data of different users are treated equally over the effect of fading channels. Hence, channel estimation and equalization for recovering the original signal of each user must be performed from cluster to cluster.

Pilot-based channel estimation is widely used in OFDM transmission system. By scattering known data called pilots into OFDM symbol at the transmitter, calculating channel values at pilot positions and then interpolating the whole channel values for data subcarriers at the receiver, transmitted information can be recovered. There are many techniques reported for channel estimation; some conventional methods like linear and cubic spline [5-8] interpolations are commonly used due to their low complexity for practical implementation, yet low efficiency. Other methods like transform-domain processing $[9,10]$ perform better but require higher computation for executing DFT/IDFT. Theoretical optimum method like minimum mean square error estimator (MMSEE) [11-15] gives best performance but is too complicate for practical realization.

Preamble is a special OFDM symbol transmitted at the beginning of transmission frame. Since it contains lots of 


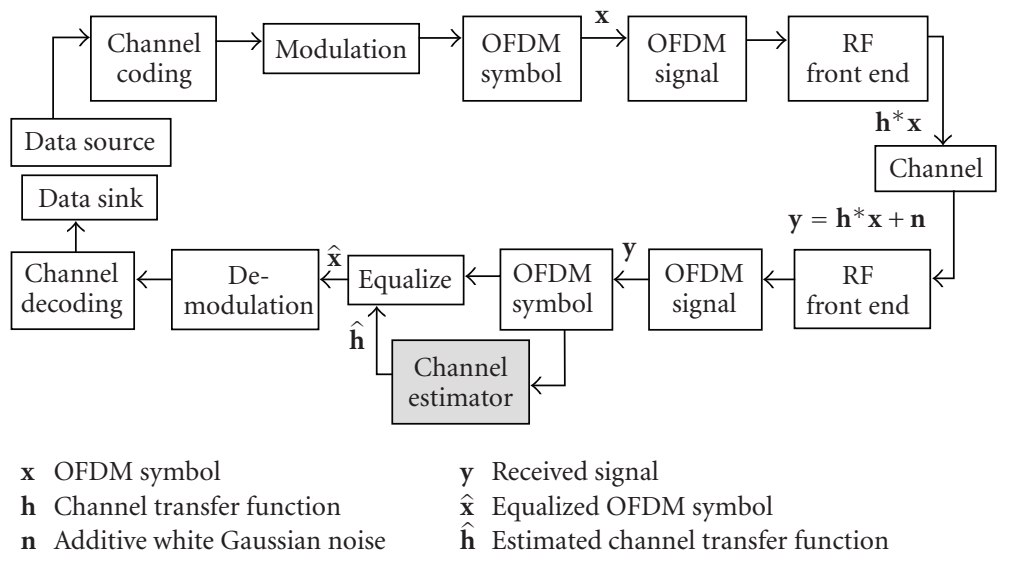

FIgure 1: An overview of OFDM system.

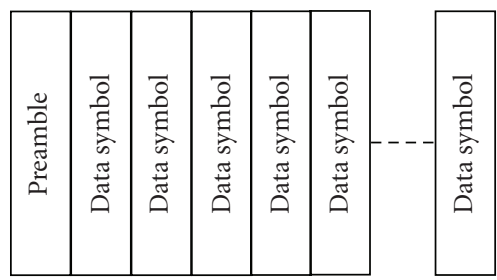

FIGURE 2: Basic transmission frame.

pilots usually evenly distributed, channel estimation task for preamble symbol is quite easy and accurate. Moreover, as the radio channel is often slowly faded, by some tracking algorithm, the estimated channel can be exploited to enhance the performance of estimation for the subsequent data symbols.

In this paper, a new scheme of channel estimation for DL-PUSC system is proposed. This approach uses LSLF technique combined with channel tracking to form a joint channel estimation scheme. Comparisons between this new method and other conventional approaches such as linear and cubic spline interpolations by simulating a typical 1024point FFT system profile in different ITU mobile channel models are given to show that a significant improvement in system performance can be achieved.

The following parts of this paper are organized as follows. Section 2 addresses the transmission structure and the signal model. Channel estimation methods such as the commonly used linear and cubic spline interpolations and the new method using LSLF technique are highlighted in Section 3. Joint scheme with tracking algorithm is introduced in Section 4. Simulation setup, results, and discussion are given in Section 5. Finally, Section 6 summarizes and concludes the paper.

\section{System Description}

2.1. Transmission Structure. A basic transmission structure is shown in Figure 2 in which a preamble symbol starts the frame and data symbols are transmitted right after.

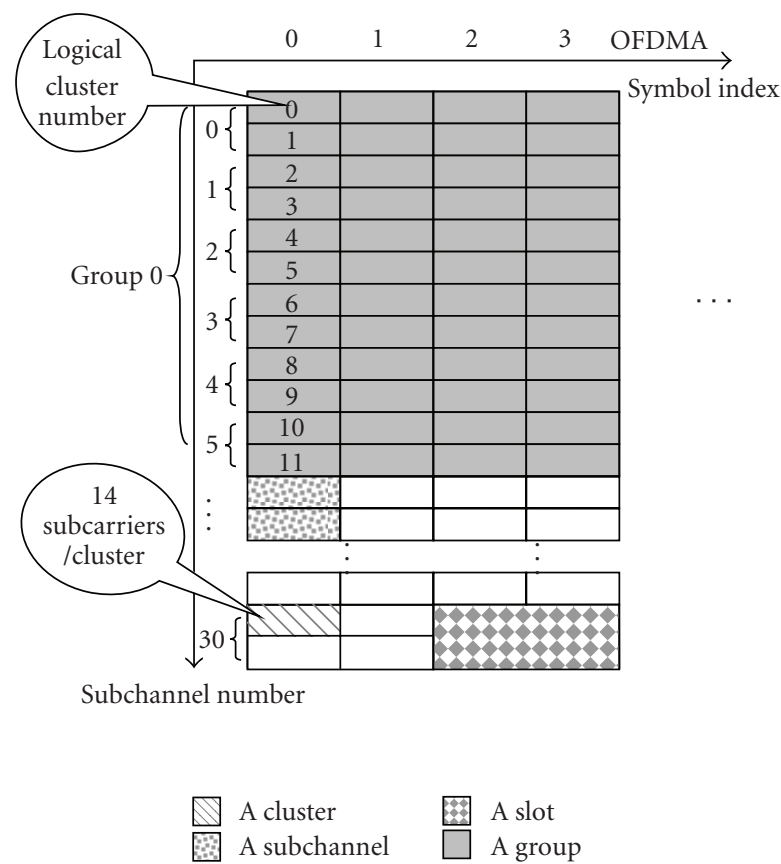

Figure 3: Basic elements in DL-PUSC mode.

Preamble symbol is designed according to different profiles of transmission, that is, in 1024-point FFT mode, there are 284 boosted BPSK pilots, each every three subcarriers, starting from subcarrier index 86 (indexing starts from 0 ). Subcarriers at other positions are set to 0. Pilot values are generated by a particular Pseudo-Noise code related to IDCell and segment parameters [1].

In DL-PUSC mode, an OFDM symbol is divided into subchannels; each of those is associated to a specific user. Subchannel is further partitioned into clusters; each of which contains a group of 14 consecutive subcarriers. When transmitted, clusters of different users will be permuted among themselves; therefore, they are scattered over the OFDM symbol. The data symbol structure is shown in Figure 3. 


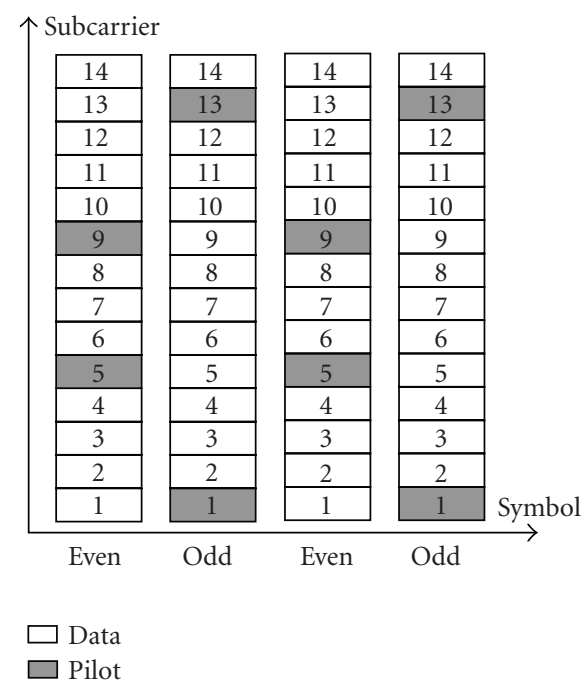

Figure 4: Pilot pattern of data symbol in a cluster.

Pilot pattern of a cluster in a DL-PUSC frame of data symbols is shown in Figure 4. Pilots are allocated at subcarrier $\{5,9\}$ for even symbol and at subcarrier $\{1,13\}$ for odd symbol.

2.2. Signal Model. Assume that transmitted frame has $M$ OFDM data symbols in which $\mathbf{x}_{m}=\left(x_{0, m}, x_{1, m}, x_{2, m}, \ldots\right.$, $\left.x_{N-1, m}\right)^{T} 0<m<M-1$ is the symbol at time $m$ and $N$ is the number of subcarriers in OFDM symbol.

At the receiver, if intersymbol interference is negligible, received signal could be derived as

$$
\mathbf{y}_{m}=\mathbf{A} \mathbf{h}_{m}+\mathbf{w}_{m},
$$

where $\mathbf{A}$ is the $N \times N$ diagonal matrix whose values are $\mathbf{x}_{m}$. The channel frequency response $\mathbf{h}_{m}=\mathbf{F g}_{m}(t)$ is the DFT of the time-varying multipath fading channel impulse response $\mathbf{g}_{m}(t)$ of which a discrete-time version can be obtained as in $[9,10] . \mathbf{F}$ is the $N \times L$ matrix whose entries are $f_{n, l}=$

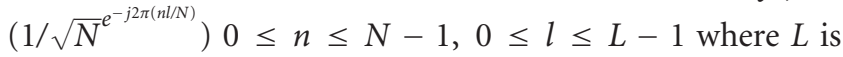
the number of channel impulse response taps, and $\mathbf{w}_{m}$ is the additive white Gaussian noise.

In order to recover $\mathbf{x}_{m}$ from $\mathbf{y}_{m}$, the channel $\mathbf{h}_{m}$ has to be estimated by exploiting the pilots which are located at predefined positions in OFDM symbols. The least-square values of channel frequency response at for pilots are obtained by

$$
h_{n_{p}, m}^{\mathrm{LS}}=\frac{y_{n_{p}, m}}{\mathbf{x}_{n_{p}, m}},
$$

where $n_{p}$ denotes the pilot position (in this particular case, $\left.n_{p}=\{1,5,9,13\}, p=0, \ldots, 3\right)$.

The goal is to estimate all the channel values $\mathbf{h}_{m}^{\mathrm{EST}}$ at all data subcarriers from the values of $\left\{h_{n_{p}, m}^{\mathrm{LS}}\right\}$ so that $\mathbf{h}_{m}^{\mathrm{EST}}$ should be as much similar as possible to $\mathbf{h}_{m}$.
Here, we have two kinds of pilot pattern depending on whether they belong to preamble symbol or data symbol. It is obvious that the density of pilot subcarriers in preamble symbol is higher than that in data symbol. Thus, in order to estimate the whole channel to have a reference for channel tracking, conventional method like linear interpolation can be utilized to get a good tradeoff between complexity and performance. On the other hand, for data symbol, in this particular case of DL-PUSC, the interpolation task must be performed from cluster to cluster, and because each cluster contains only 14 consecutive subcarriers, the channel on each cluster can be approximated as a "line"; this fact inspires the idea of using LSLF technique to estimate the partial channel. Therefore, a comparative study is carried out to demonstrate the superiority of this approach to other commonly used methods such as linear $[5,6]$ and cubic spline $[7,8]$ interpolations.

Due to the pilot arrangement in data symbols, it is necessary to perform a two-dimension (2D) interpolation scheme or two 1D estimations in cascade. As the number of pilots in time axis is more than that in frequency axis, it is more convenient to estimate first in time and then in frequency.

\section{Channel Estimation}

\subsection{Conventional Methods}

3.1.1. Linear Interpolation. Figure 5 shows an example of the linear interpolation technique in time direction for an even number of OFDM symbols in which pilots of even symbols are located at the 5th and 9th locations while those of odd symbols are resided in the 1 st and 13 th places. Consider

$$
\begin{gathered}
h_{\{1,13\}, m}^{\mathrm{EST}}= \begin{cases}h_{\{1,13\}, 1}^{\mathrm{LS}}, & m=0, \\
\frac{h_{\{1,13\}, m-1}^{\mathrm{LS}}+h_{\{1,13\}, m+1}^{\mathrm{LS}}}{2} & m=2,4, \ldots, M-2,\end{cases} \\
h_{\{5,9\}, m}^{\mathrm{EST}}= \begin{cases}\frac{h_{\{5,9\}, m-1}^{\mathrm{LS}}+h_{\{5,9\}, m+1}^{\mathrm{LS}},}{2} & m=1,3, \ldots, M-3, \\
h_{\{5,9\}, M-2}^{\mathrm{LS}}, & m=M-1 .\end{cases}
\end{gathered}
$$

Then, interpolation in frequency direction can be evaluated as

$$
\begin{aligned}
& h_{k, m}^{\mathrm{EST}}=k \Delta+h_{n_{\mathrm{p}}, m}^{\mathrm{LS} / \mathrm{EST}}, \quad k=1, \ldots, 4, \\
& \Delta=\frac{h_{n_{p+1}, m}^{\mathrm{LS} / \mathrm{EST}}-h_{n_{p}, m}^{\mathrm{LS} / \mathrm{EST}}}{4}, \quad p=0,1,2,
\end{aligned}
$$

where $k$ denotes the position of channel value inside the interval of two adjacent pilots $\left(h_{n_{p}, m}^{\mathrm{LS} / \mathrm{EST}}, h_{n_{p+1}, m}^{\mathrm{LS} / \mathrm{EST}}\right)$.

3.1.2. Cubic Spline Interpolation. With this method, because we do not have enough pilots in frequency direction, interpolation in time has to be performed first. Moreover, it also requires having at least 8 OFDM symbols to have 


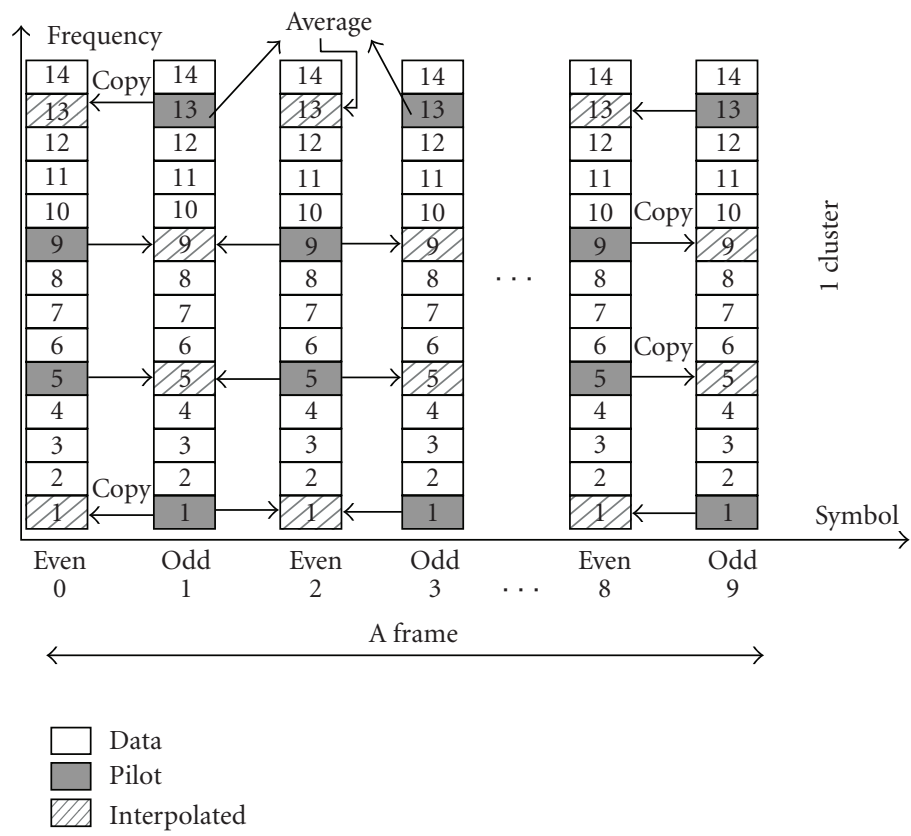

Figure 5: Linear interpolation in time.

enough pilot points. Parameters of a third-ordered equation are calculated as indicated in [8] and [16]. Then, the channel values at data positions within the appropriate interval are calculated. After interpolation in time, there are enough points to carry out this task again in frequency direction.

3.2. Channel Estimation Using LSLF. Since a cluster size is small, channel transfer function of that cluster can be considered a "line". Therefore, the least-squares line that fits all the pilots is thought to be a better approximation of the ideal channel.

3.2.1. Interpolation in Time. Suppose that an even number of OFDM symbols appears in frame and is indexed from 0 to $M-1$. In time direction, for a cluster, there are four sub-streams according to subcarrier indexes $\{1,5,9,13\}$ containing channel values at pilots. Stream 1 and stream 13 have pilots at odd locations $\{1,3,5, \ldots, M-1\}$ while stream 5 and stream 9 have pilots at even locations $\{0,2,4, \ldots, M-2\}$. Define the channel values at pilot positions vector $\mathbf{p}=\left\{p_{k}\right\}$ and pilot position vector $\mathbf{l}=\left\{l_{k}\right\},(k=1,2, \ldots, M / 2)$ in which $\mathbf{l}=(0,2, \ldots, M-2)$ for stream $\{5,9\}$ and $\mathbf{l}=$ $(1,3, \ldots, M-1)$ for stream $\{1,13\}$; LSLF technique will find the pair of coefficients $\omega=\left(\begin{array}{l}a \\ b\end{array}\right)$ to form the line containing the set of points: $\mathbf{y}=\left\{y_{k}\right\} ; y_{k}=a l_{k}+b$ so that the leastsquares error

$$
s=\sum_{k=1}^{M / 2}\left(p_{k}-y_{k}\right)^{2}=\sum_{k=1}^{M / 2}\left(p_{k}-a l_{k}-b\right)^{2}
$$

is minimized. That means to find a pair of coefficients $\{a, b\}$ so that they minimize $s$ and so vanish the partial derivatives $(\partial s / \partial a)$ and $(\partial s / \partial b)$. Therefore, from [17], problem turns into solving this system of equations

$$
\begin{aligned}
\frac{\partial s}{\partial a} & =0, \\
\frac{\partial s}{\partial b} & =0 \\
& \Leftrightarrow\left\{\begin{array}{l}
a=\frac{(M / 2) \sum_{k=1}^{M / 2} l_{k} p_{k}-\sum_{k=1}^{M / 2} l_{k} \sum_{k=1}^{M / 2} p_{k}}{(M / 2) \sum_{k=1}^{M / 2} l_{k}^{2}-\left(\sum_{k=1}^{M / 2} l_{k}\right)^{2}}, \\
b=\frac{\sum_{k=1}^{M / 2} l_{k}^{2} \sum_{k=1}^{M / 2} p_{k}-\sum_{k=1}^{M / 2} l_{k} \sum_{k=1}^{M / 2} l_{k} p_{k}}{(M / 2) \sum_{k=1}^{M / 2} l_{k}^{2}-\left(\sum_{k=1}^{M / 2} l_{k}\right)^{2}} .
\end{array}\right.
\end{aligned}
$$

Channel values at all locations including data and pilots in stream $1,5,9$, and 13 will be calculated by applying

$$
h_{k^{\prime}}^{\mathrm{dataEST}}=a l_{k^{\prime}}+b, \quad k^{\prime}=0,1, \ldots, M-1 .
$$

It is important to derive the maximum number of OFDM symbols $M$ so that the fitting by using a "line" is reasonable. By the fact that the fading channel will change in time with a coherent time $T_{c}$ as mentioned deeper in next section, it is clear to see that the limit should be $M T_{\text {symbol }}<T_{c}$. So a rough limit range for $M$ can be $4 \leq M<T_{c} / T_{\text {symbol }}$ and for convenient $M$ should be even number.

3.2.2. Interpolation in Frequency. The same routine as in time axis can be used to interpolate in frequency axis. Now, there is a block of $M$ clusters; each cluster contains 14 subcarriers in which 4 locations were estimated values from the previous task. One note is that all clusters now have "pilots" at the same indexes; hence, the complexity is less. 
TAble 1: Profile parameters.

\begin{tabular}{lccc}
\hline Bandwidth & $8.75 \mathrm{MHz}$ & FFT size & 1024 \\
\hline Sampling factor $n$ & $8 / 7$ & Number of used subcarriers $N$ used & 840 \\
Sampling frequency & $10 \mathrm{MHz}$ & Frame structure & 1 preamble symbol + 48 data symbol \\
Subcarrier space & $9.765625 \mathrm{KHz}$ & Modulation mode & QPSK 16-QAM 64-QAM \\
Useful symbol time $\mathrm{Tb}$ & $102.4 \mu \mathrm{s}$ & CP ratio G & $1 / 8$ \\
Guard interval $\mathrm{Tg}$ & $12.8 \mu \mathrm{s}$ & Channel coding & CC $(171,133) \mathrm{rate} 1 / 2$ \\
OFDM symbol time $T s$ & $115.2 \mu \mathrm{s}$ & Carrier frequency & $2.3 \mathrm{GHz}$ \\
Number of user & 3 & System mode & DL-PUSC \\
\hline
\end{tabular}

TABLE 2: Profiles of channels used in simulation.

\begin{tabular}{|c|c|c|c|c|c|c|c|}
\hline \multirow[t]{2}{*}{ Model 1 Ped.B } & Path power $(\mathrm{dB})$ & -3.9 & -4.8 & -8.8 & -11.9 & -11.7 & -27.8 \\
\hline & Path delay $(\mu \mathrm{s})$ & 0 & 0.2 & 0.8 & 1.2 & 2.3 & 3.7 \\
\hline \multirow[t]{2}{*}{ Model 2 Veh.A } & Path power $(\mathrm{dB})$ & -3.1 & -4.1 & -12.1 & -13.1 & -18.1 & -23.1 \\
\hline & Path delay $(\mu \mathrm{s})$ & 0 & 0.31 & 0.71 & 1.09 & 1.73 & 2.51 \\
\hline
\end{tabular}

Again, it is crucial to judge the assumption that channel transfer function in a cluster can be viewed as line. The fact that the frequency range of 14 subcarriers or one cluster should be less than the coherent bandwidth $B_{c}$ of the fading channel [18] gives $B_{c} \approx 1 / 5 \sigma_{\tau}$ in which $\sigma_{\tau}$ denotes the root mean squared delay spread of the multipath fading channel. Another factor is the bandwidth of the designed system; the performance of this method would degrade when system bandwidth is significantly broader than $B_{c}$ so that a small portion as cluster could also be frequency selective.

\section{Joint Scheme with Channel Tracking}

For preamble symbol, the least-square channel values at pilot positions are

$$
h_{n_{p}}^{\mathrm{pre}^{\mathrm{LS}}}=\frac{y_{n_{p}}^{\mathrm{pre}}}{x_{n_{p}}^{\mathrm{pre}}} .
$$

In the case of DL-PUSC mode, pilots in preamble symbol are evenly spaced scattered, one every three subcarriers. The whole channel values can be linearly interpolated (except the two zero-guard interval regions) as

$$
h_{n_{p}+i}^{\mathrm{preEST}}=\left(\frac{L-i}{L}\right) h_{n_{p}}^{\mathrm{pre}}+\left(\frac{i}{L}\right) h_{n_{p+1}}^{\mathrm{preLS}}
$$

where $i=1,2 ; L=3 ; n_{p}=86+3 p ; p=0, \ldots, 282$.

In fact, the channel does not stay the same over time but slowly changes; this is due to the relative movement of all the components influencing the transmission. The most impact factor is the relative speed between mobile station and base station that causes a Doppler frequency shift $f_{D}$. The coherent time $T_{c} \approx 1 / f_{D}$ over which the channel can be viewed as unchanged is in the order of several milliseconds to hundreds of milliseconds. Hence, it is considered "slow" when comparing to an OFDM symbol time slot. As a result, after estimating the whole channel from the preamble symbol, performance of channel estimation for successive data symbols can be enhanced by a tracking algorithm [9].
Suppose that $h_{n, m}^{\text {dataEST }}$ is the estimated channel value at subcarrier $n$ of data symbol $m$; it can be recalculated so that some useful information from the preamble symbol can be involved to reduce the distance between it and the real channel, and thus enhance the estimation performance:

$$
\hat{h}_{n, m}^{\text {data }}=\left(\frac{M-m-1}{M}\right) h_{n}^{\text {preEST }}+\left(\frac{m+1}{M}\right) h_{n, m}^{\text {dataEST }},
$$

where $n=0, \ldots, N-1 ; m=0, \ldots, M-1$.

This tracking algorithm means that the nearer the data symbol is located to the preamble symbol; the more influent it gets from the estimated channel given by the preamble and vice versa.

\section{Simulation Results}

5.1. Simulation Setup. The typical 1024-point FFT profile, whose parameters are given in Table 1, is chosen for simulation. The number of users requesting service is assumed to be 3 .

Channel models are taken from ITU models for mobile environments [19], and carrier frequency is set to be $2.3 \mathrm{GHz}$ :

(i) Model 1: ITU_Pedestrian B (Ped.B), speed $6 \mathrm{~km} / \mathrm{h}$, and fading frequency $f_{D} \approx 12.78 \mathrm{~Hz}$,

(ii) Model 2: ITU_Vehicular A (Veh.A), speed $30 \mathrm{~km} / \mathrm{h}$ with $f_{D} \approx 63.89 \mathrm{~Hz}$, and speed $120 \mathrm{~km} / \mathrm{h}$ with $f_{D} \approx$ $255.56 \mathrm{~Hz}$.

These channel models are time-variant frequency selective channels in Non Line-Of-Sight mobile conditions. Their specific parameters are given in Table 2.

System performance is demonstrated as packet error rate (PER) versus signal-to-noise ratio (SNR) and user link throughput defined as

$$
T=D(1-\text { PER })
$$

where $D$ is the peak data rate given by

$$
D=N_{s} N_{b} R_{c} / T_{s}
$$




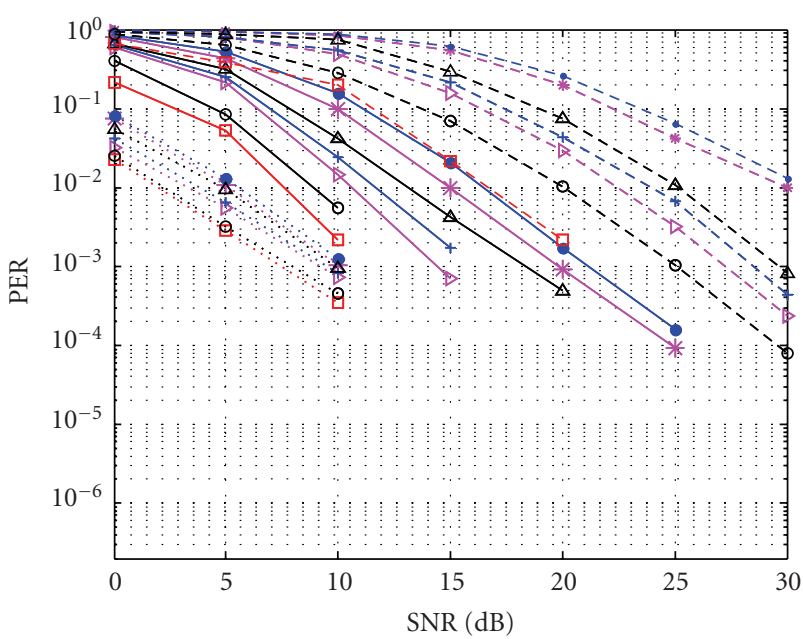

$\begin{array}{lcl}\square \text { Perfect channel knowledge } & \Delta & \text { LSLF method } \\ \text { * Linear interpolation } & \circ & \text { LSLF method \& tracking } \\ \triangleright \text { Linear interpolation \& tracking } & --- \text { QPSK } \\ \text { - Spline interpolation } & - & \text { 16-QAM } \\ + \text { Spline interpolation \& tracking } & \cdots \cdots & \text { 64-QAM }\end{array}$

Figure 6: PERs in Ped.B $6 \mathrm{~km} / \mathrm{h}$.

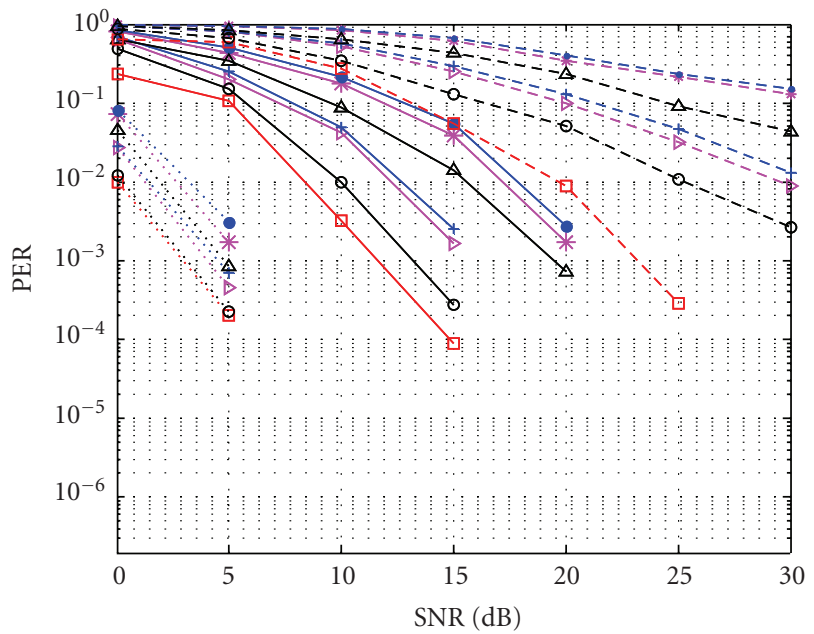

口 Perfect channel knowledge

* Linear interpolation

$\triangleright$ Linear interpolation \& tracking

- Spline interpolation

+ Spline interpolation \& tracking

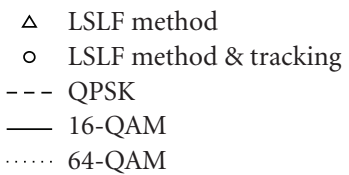

Figure 7: PERs in Veh.A $30 \mathrm{~km} / \mathrm{h}$.

in which $N_{s}, N_{b}, R_{c}$, and $T_{s}$ denote the number of subcarriers assigned to a user, number of data bits in a subcarrier, channel coding rate, and the OFDM symbol time, respectively.

5.2. Simulation Results and Discussion. Simulation results are shown in Figure 6 to Figure 14. The very first notice is that in all channel conditions the LSLF approach always outperforms the other two conventional methods. The

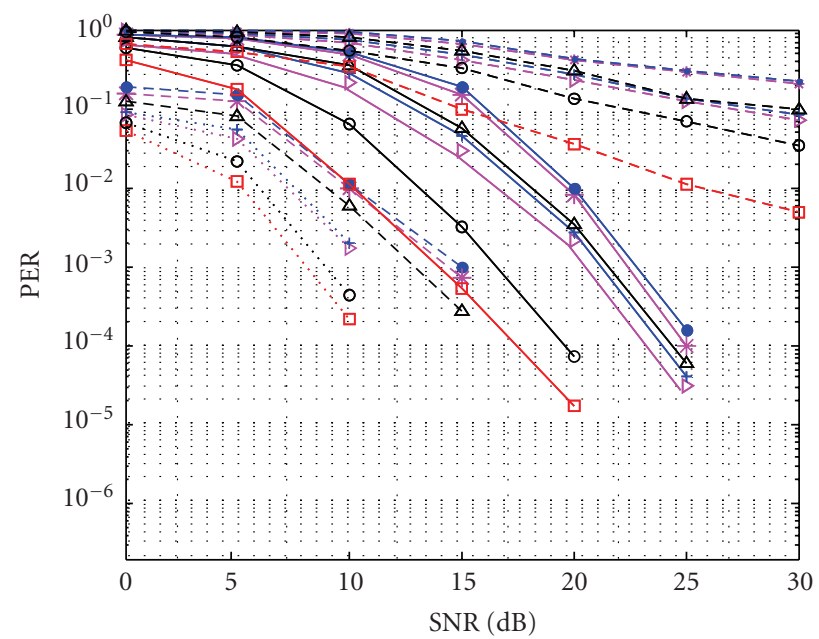

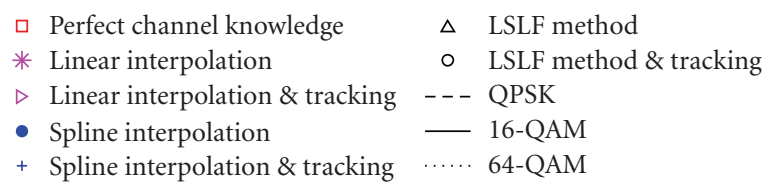

Figure 8: PERs in Veh.A $120 \mathrm{~km} / \mathrm{h}$.

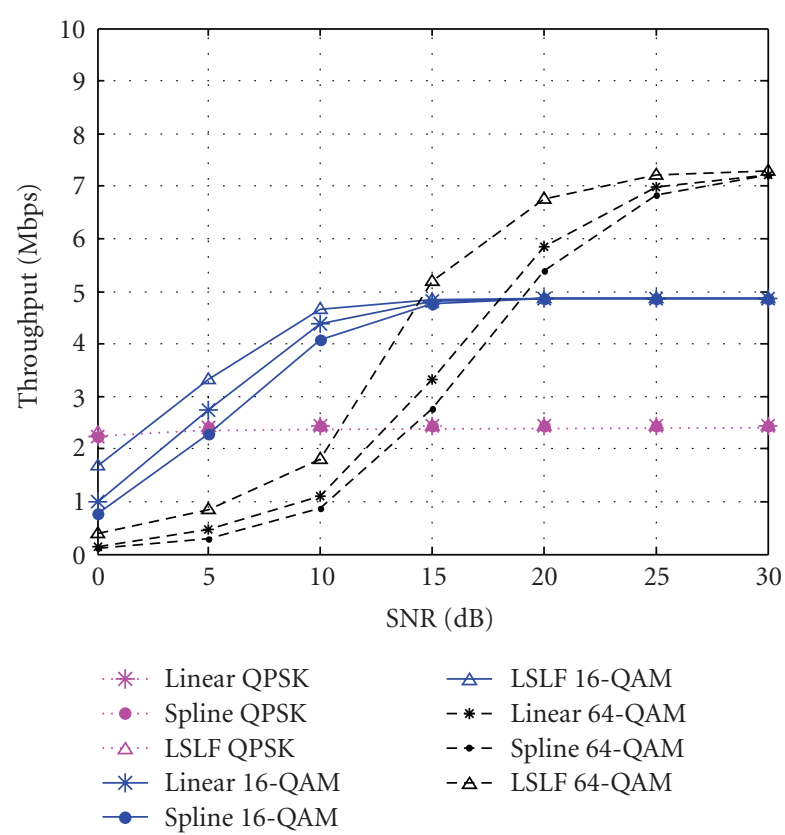

Figure 9: Throughput in Ped.B $6 \mathrm{~km} / \mathrm{h}$ without using channel tracking.

improvement varies depending on which modulation mode is used. It is also very clear to see that when channel tracking comes into play, regardless of modulation schemes and channel conditions, the performance is remarkably boosted. The joint scheme of LSLF channel estimation and channel tracking appears to be the best, very robust, and not only highly surpassing other schemes but also able to reach very near to the perfect channel knowledge case. 


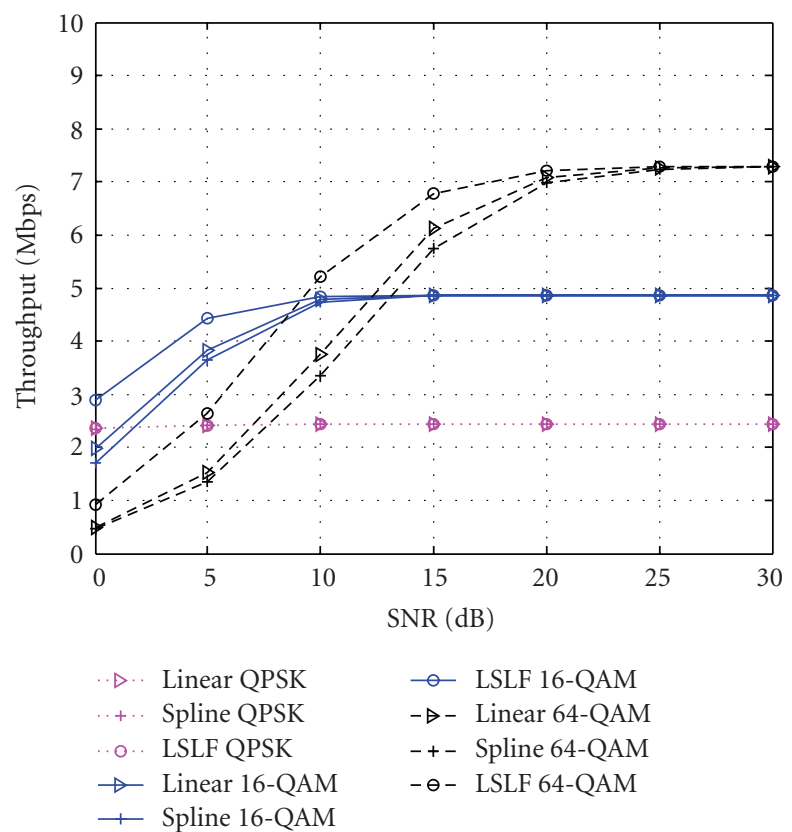

Figure 10: Throughput in Ped.B $6 \mathrm{~km} / \mathrm{h}$ using channel tracking.

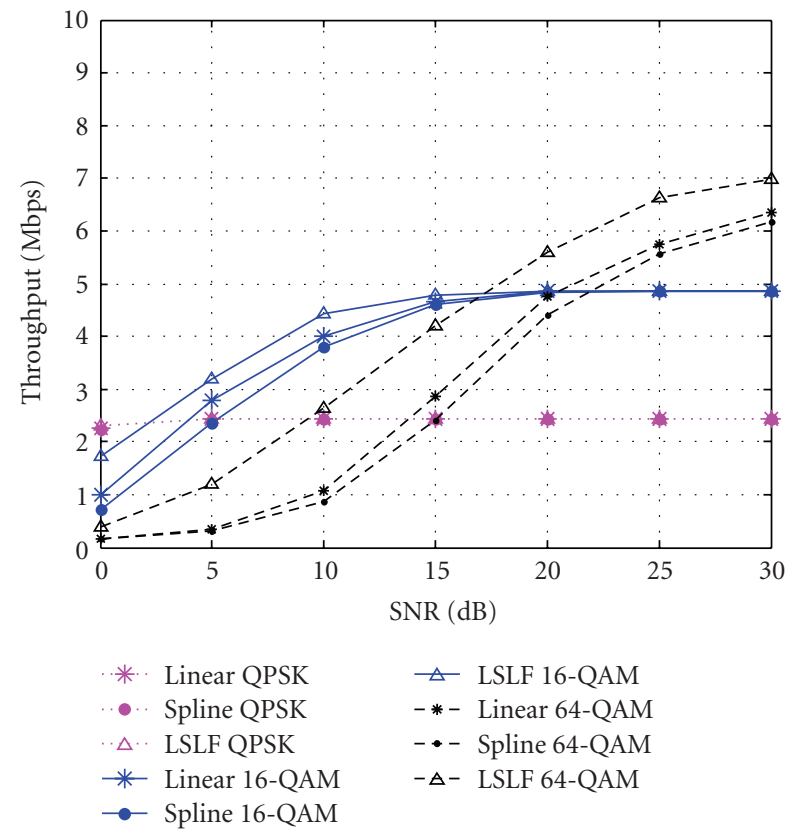

FIgUre 11: Throughput in Veh.A $30 \mathrm{~km} / \mathrm{h}$ without using channel tracking.

Figures 6, 7, and 8 show PERs in different channel models. Ped.B channel has quite long delay spread, causing severely frequency-selective faded channel and limiting the performance of channel estimation, particularly in frequency axis. However, due to the slow moving speed, the channel does not change rapidly, giving some favor to estimation in time. One can notice that the effect given by channel tracking in this channel is not as strong as that in Veh.A channels.

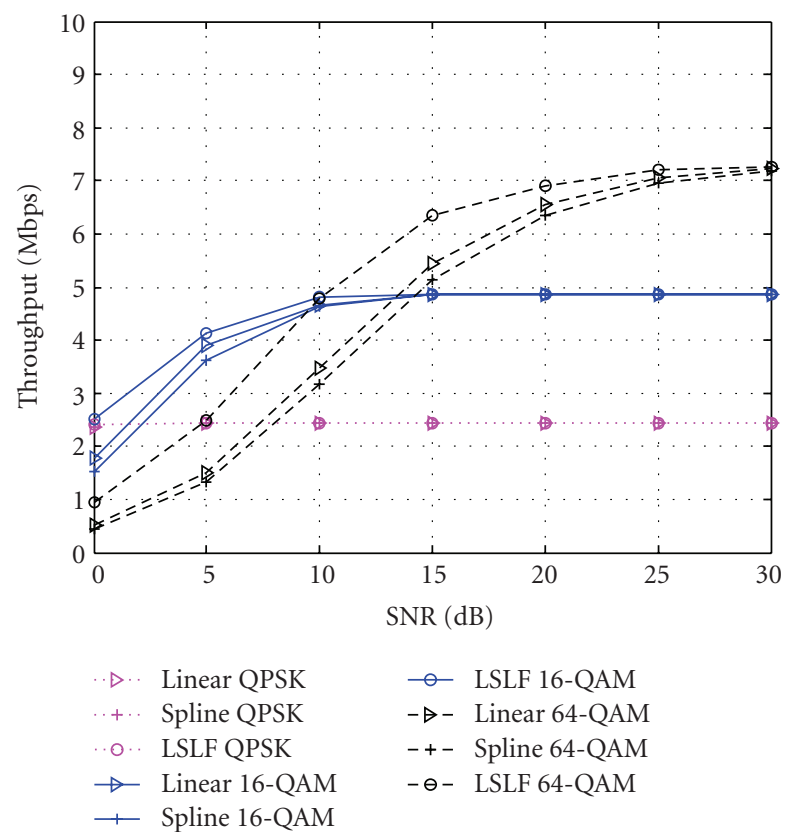

FIgure 12: Throughput in Veh.A $30 \mathrm{~km} / \mathrm{h}$ using channel tracking.

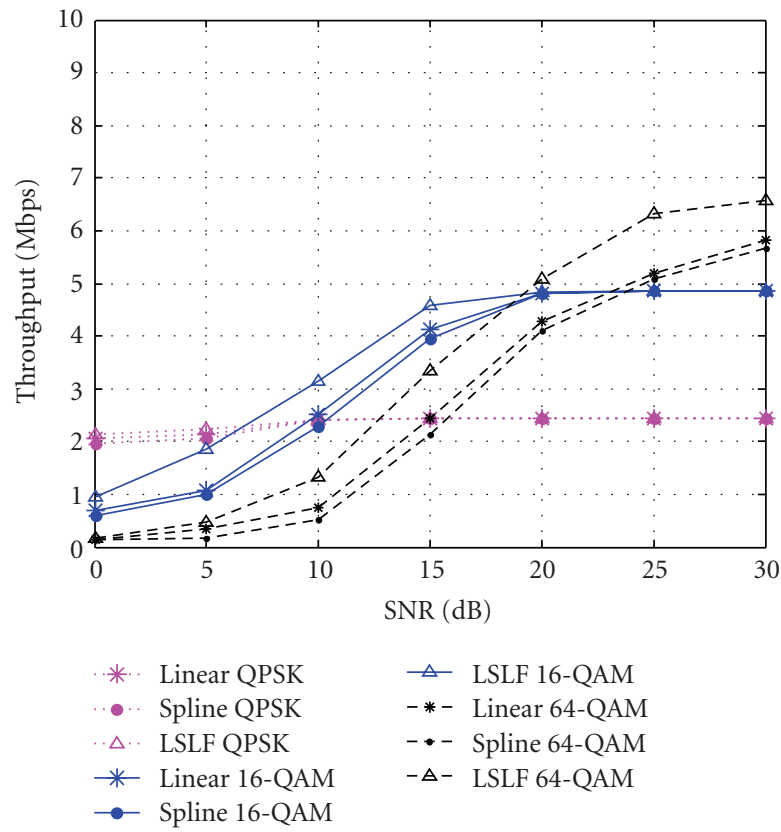

FIGURE 13: Throughput in Veh.A $120 \mathrm{~km} / \mathrm{h}$ without using channel tracking.

On the other hand, Veh.A channels have smaller delay spread but higher moving speed, meaning that the channel within a cluster is flat but it changes faster. The coherent time of this channel in case of speed $120 \mathrm{~km} / \mathrm{h}$ is in order of several milliseconds which can degrade the system performance since it might go below the frame time. However, the LSLF still works properly and with channel tracking; at least 5-dB enhancement can be achieved. 


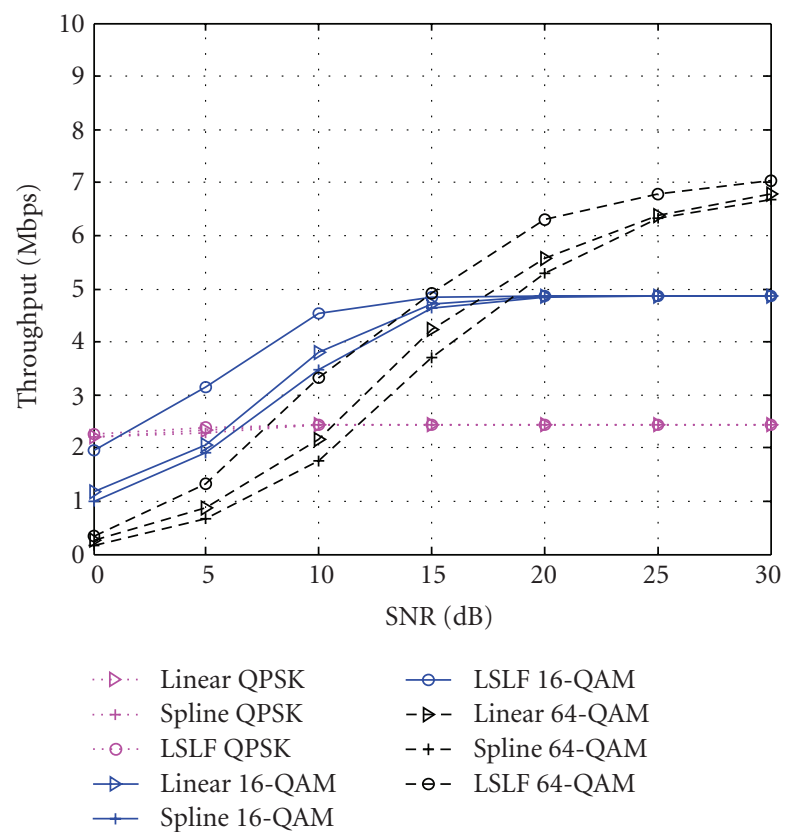

FIgURE 14: Throughput in Veh.A $120 \mathrm{~km} / \mathrm{h}$ using channel tracking.

Figures 9, 10, 11, 12, 13, and 14 show the user link throughputs in various channel conditions without and with channel tracking. Obviously, channel tracking give a noticeable improvement and the joint scheme of LSLF channel estimation with tracking significantly increases the link performance.

Another notice is that the performance improvement also depends on modulation modes. The higher modulation modes always suffer higher error in channel estimation, leading to more degradation compared to the perfect channel knowledge case whereas in lower modulation mode, for example, QPSK, the joint scheme is able to reach the ideal case.

Last but not least, it is worth to examine roughly the complexity of LSLF method for practical implementation. From equations (7), it is obvious that the LSLF method needs more computation than linear and cubic spline interpolation but it does not require any complicated process or special design structure. There is no complex operation since the inphase and quadrature components can be treated separately whereas there are also some terms in (7) that can be reused. Therefore, the superior performance gain obtained by the joint scheme with channel tracking makes this method very promising for realization.

\section{Conclusions}

This paper has studied a joint channel tracking and estimating scheme which is highly suitable for OFDMA DL-PUSC mode of mobile WiMAX system. System simulation with various standardized channel models for mobile environments showed impressive improvements in both PER and user link throughput. Low complexity and high performance give this joint scheme a high potential for practical implementation.

\section{References}

[1] "IEEE Standard for Local and Metropolitan area networks Part 16," The Institute of Electrical and Electronics Engineering, Inc. Std. IEEE 802.16e, 2005.

[2] "IEEE Standard for Local and Metropolitan area networks Part 16," The Institute of Electrical and Electronics Engineering, Inc. Std.IEEE 802.16d, 2004.

[3] G. Parsaee and A. Yarali, "OFDMA for the 4th generation cellular networks," in Proceedings of the Canadian Conference on Electrical and Computer Engineering (CCECE '04), vol. 4, pp. 2325-2330, 2004.

[4] O. Edfors, M. Sandell, J.-J. Van de Beek, D. Landström, and F. Sjöberg, An Introduction to Orthogonal Frequency Division Multiplexing, Luleå Tekniska Universitet, Luleå, Sweden, 1996.

[5] Y. Shen and E. F. Martinez, "WiMAX channel estimation: algorithms and implementations," Tech. Rep. AN3429, Freescale Semiconductor Inc., Brooklyn, NY, USA, 2007.

[6] Y. Shen and E. F. Martinez, "Channel estimation in OFDM systems," Tech. Rep. AN3059, Freescale Semiconductor Inc., Brooklyn, NY, USA, 2006.

[7] M. Henkel, C. Schilling, and W. Schroer, "Comparison of channel estimation methods for pilot aided OFDM systems," in Proceedings of the IEEE Vehicular Technology Conference (VTC '07), pp. 1435-1439, Dublin, Ireland, April 2007.

[8] S. Coleri, M. Ergen, A. Puri, and A. Bahai, "A study of channel estimation in OFDM systems," in Proceedings of the 65th IEEE Vehicular Technology Conference (VTC '02), vol. 56, no. 2, pp. 894-898, 2002.

[9] X. Dong, X. Xie, and X. Chen, "Joint channel estimation for WiMAX by preamble and uneven pilot," in Proceedings of the International Conference on Wireless Communications, Networking and Mobile Computing (WiCOM '07), pp. 11041107, September 2007.

[10] Y. Zhao and A. Huang, "A novel channel estimation method for OFDM mobile communication systems based on pilot signals and transform-domain processing," in Proceedings of the 47th IEEE Vehicular Technology Conference (VTC '97), vol. 3, pp. 2089-2093, May 1997.

[11] J.-J. van de Beek, O. Edfors, M. Sandell, S. Wilson, and P. Borjesson, "On channel estimation in OFDM systems," in Proceedings of the IEEE Vehicular Technology Conference (VTC '95), vol. 2, pp. 815-819, Chicago, Ill, USA, July 1995.

[12] S. Coleri, M. Ergen, A. Puri, and A. Bahai, "Channel estimation techniques based on pilot arrangement in OFDM systems," IEEE Transactions on Broadcasting, vol. 48, no. 3, pp. 223-229, 2002.

[13] T. Yücek, M. K. Özdemir, H. Arslan, and F. E. Retnasothie, "A comparative study of initial downlink channel estimation algorithms for mobile WiMAX," in Proceedings of the IEEE Mobile WiMAX Symposium, pp. 32-37, March 2007.

[14] M. Morelli and U. Mengali, "A comparison of pilot-aided channel estimation methods for OFDM systems," IEEE Transactions on Signal Processing, vol. 49, no. 12, pp. 3065-3073, 2001.

[15] M. Sandell and O. Edfors, "A comparative study of pilot-based channel estimators for wireless OFDM," Tech. Rep., Signal Processing Division, Luleå University of Technology, Luleå, Sweden, September 1996.

[16] E. Weisstein, Cubic Spline, The MathWorld Book, Wolfram Math World. 
[17] E. Weisstein, Least Squares Fitting, The MathWorld Book, Wolfram Math World.

[18] B. Sklar, "Rayleigh fading channels in mobile digital communication systems part I: characterization," IEEE Communications Magazine, vol. 35, no. 9, pp. 136-146, 1997.

[19] Recommendation ITU-R M.1225, “Guidelines for evaluation of radio transmission technologies for IMT-2000," 1997. 

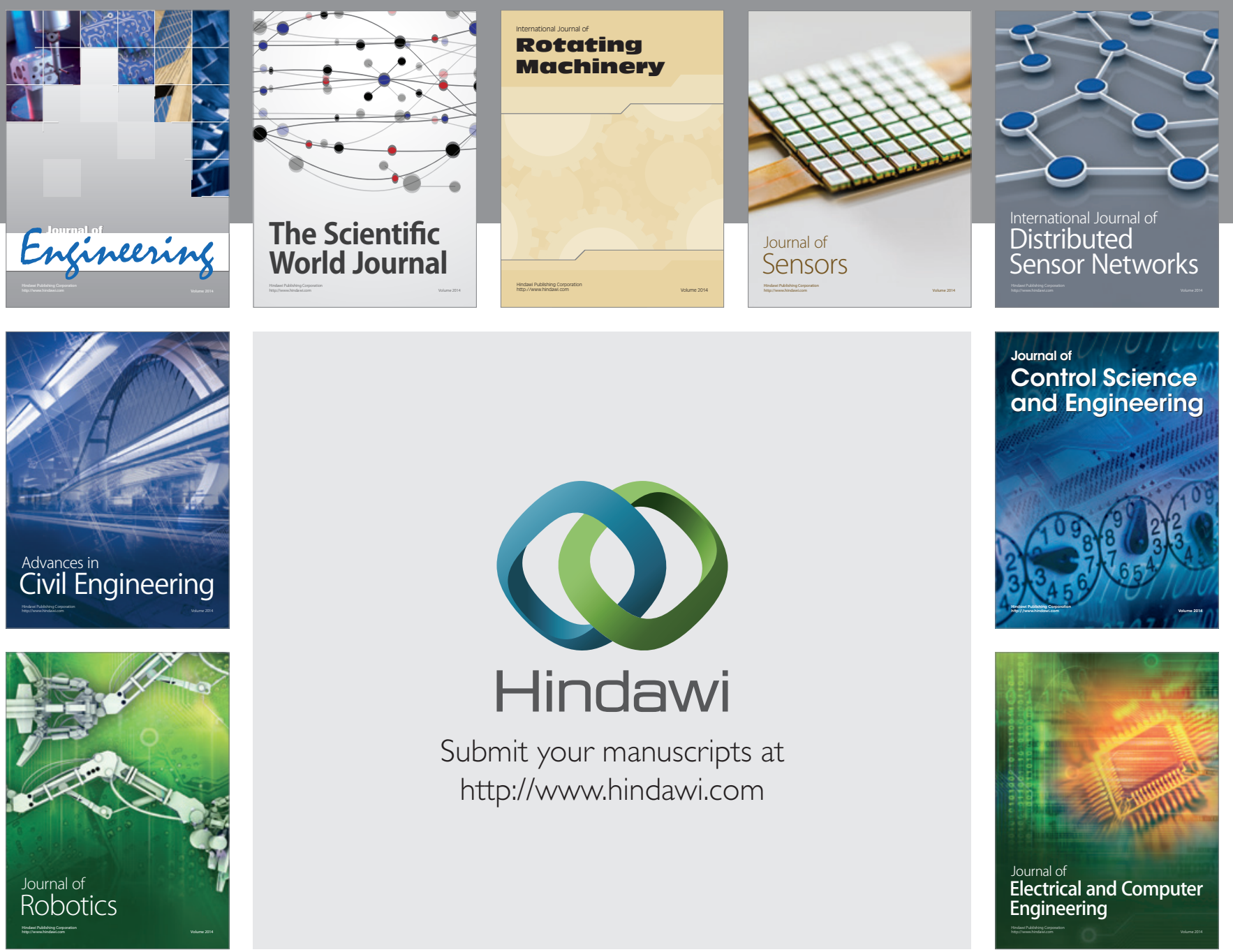

Submit your manuscripts at

http://www.hindawi.com
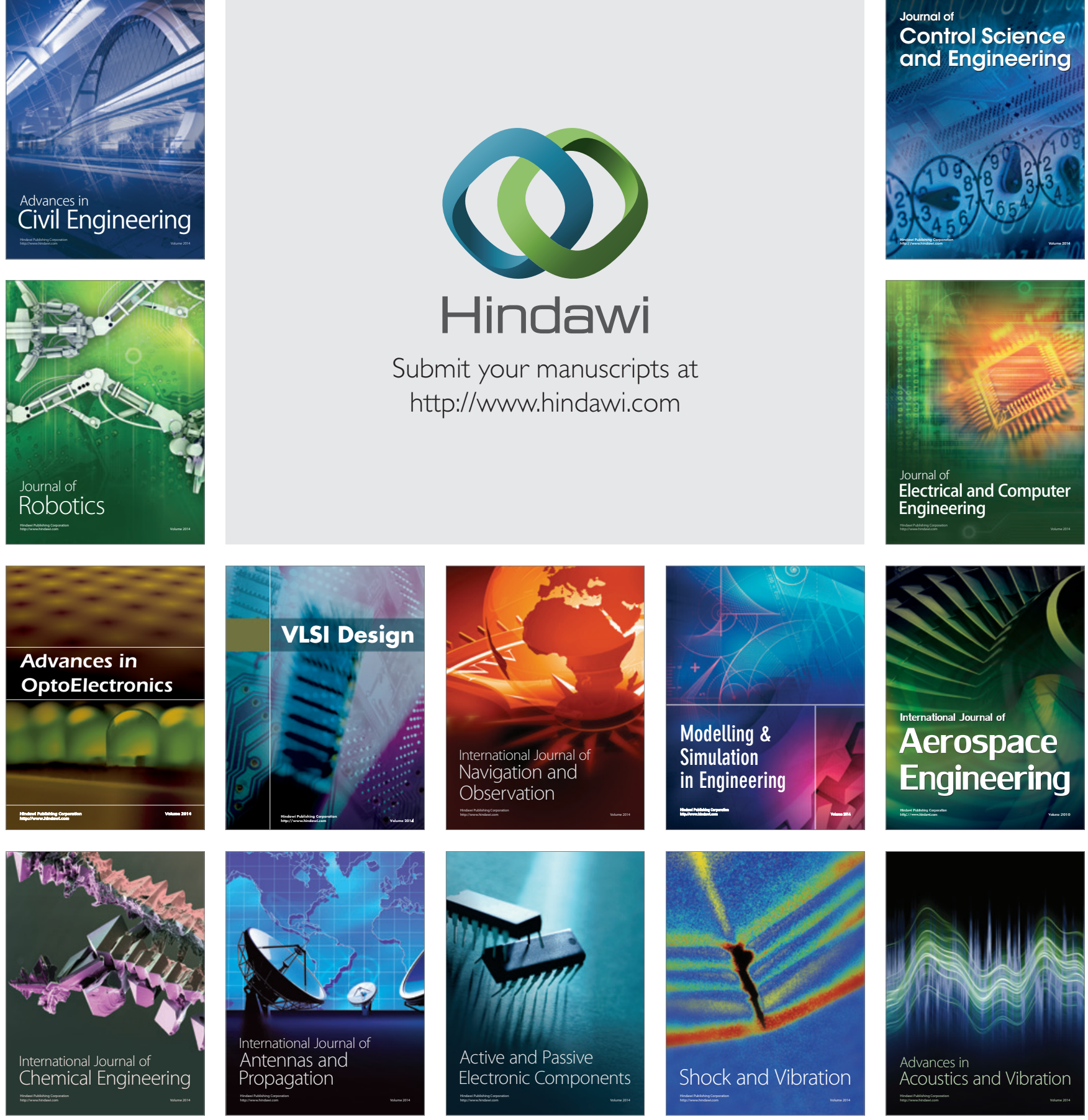